\title{
DETERMINACIÓN DEL DIMORFISMO SEXUAL SECUNDARIO EN LA GUACAMAYA VERDE (ARA MILITARIS): COMPLEMENTARIEDAD MORFOMÉTRICA E ÍNDICE OCULAR
}

\author{
Carlos Bonilla-Ruz,* Claudia Cinta Magallón \& Antonio Santos-Moreno \\ Centro Interdisciplinario de Investigación para el Desarrollo Integral Regional, unidad Oaxaca, \\ Instituto Politécnico Nacional. Calle Hornos 1003, Indeco Xoxo, Santa Cruz Xoxocotlán, Oaxaca. C.P. \\ 71230. MEXICO.*<cbonill@hotmail.com>
}

Bonilla-Ruz, C., C. Cinta Magallón \& A. Santos-Moreno. 2011. Determinación del dimorfismo sexual secundario en la guacamaya verde (Ara militaris): complementariedad morfométrica e índice ocular. Acta Zoológica Mexicana (n. s.), 27(2): 245-255.

RESUMEN. La mayor parte de los psitácidos americanos no presentan un dimorfismo sexual secundario apreciable. La distinción de los sexos permite obtener información biológica relevante para poblaciones de especies amenazadas o en peligro de extinción. En el presente trabajo se presentan los resultados de un análisis morfométrico para determinar diferencias sexuales secundarias en Ara militaris. La hipótesis a probar en nuestro análisis es que existen diferencias sexuales en esta especie, que pueden ser observables en la forma, tamaño y proporciones de ciertas partes de la cabeza. Para comprobarlo, se utilizaron 18 ejemplares en cautiverio casi todos con sexo conocido a partir de análisis de ADN (11 hembras, 4 machos y 3 de sexo desconocido). Se obtuvieron 11 medidas de diferentes partes de la cabeza a partir de fotografías de cada ejemplar. Se realizó un Análisis de Componentes Principales con el que se identificaron las medidas más significativas en la diferenciación de sexos de la especie. A partir de nuestros resultados se propone un índice ocular que discrimina más fácilmente entre sexos a partir de los datos originales. Se obtienen de esta forma dos indicadores útiles, complementarios y muy económicos para el estudio de campo, en actividades de manejo y monitoreo de esta especie y que podrían ser útiles para otras especies de psitácidos.

Palabras clave: Ara militartis, variación intrapoblacional, dimorfismo sexual secundario, determinación de sexo.

Bonilla-Ruz, C., C. Cinta Magallón \& A. Santos-Moreno. 2011. Determination of sexual secundary dimorphism in military macaw (Ara militaris): morphometry complementary with ocular index. Acta Zoológica Mexicana (n. s.), 27(2): 245-255.

ABSTRACT. Most American psittacids do not show sexual dimorphism. Finding differences between sexes allows getting practical biological information about populations of threatened or endangered species. This work shows the significant results of a morphometric study to indicate significant secondary

Recibido: 13/10/2009; aceptado: 12/01/2011. 
sexual differences on Ara militaris. The hypothesis tested on this analysis is that there are secondary sexual differences on this species that can be visually detected; we show that the size and proportions and layout of certain parts of the head can be used consistently and reliably to differentiate males from females. To test the hypothesis, we used 18 specimens in captivity, 15 of them sexed by DNA analysis (11 females, 4 males plus 3 of unknown sex). We used 11 measurements taken from pictures of the head of each specimen from different parts of the head. A Principal Components Analysis was performed and most significant measurements were identified from these results, therefore an ocular index was built. The ocular index correlates two involved characteristics in the ocular area of the bird, providing information to make an easy differentiation of the sexes. Predictions made with this ocular index matched the DNA results; the sex from the three unidentified specimens was predicted by using the index. The results represent a very practical, complementary, and inexpensive way to differentiate sex among these birds for field management and monitoring purposes.

Key Words: Ara militaris, intrapopulation variation, secondary sexual dimorphism, sex determination.

\section{INTRODUCCIÓN}

Aunque algunas especies de psitácidos presentan dimorfismo sexual secundario como es el caso del periquito enano (Forpus cyanopygius), donde la presencia de azul turquesa en las alas y en la rabadilla define al macho, o en los casos del loro frentiblanco (Amazona albifrons) y del loro de anteojos rojos (Amazona pretrei) donde la existencia de áreas rojas más extensas y conspicuas son características del macho (Forshaw 1978, Juniper \& Parr 1998), desde el punto de vista sexual secundario los psitácidos son predominantemente monomórficos, y se considera que el $75 \%$ de las especies, entre ellas Ara militaris, no presentan dimorfismo sexual (Santos et al. 2006); machos y hembras son indistinguibles externamente unos de otros. Para conocer el sexo de los individuos de especies monomórficas existen métodos invasivos y no invasivos; dentro de los primeros sobresale el examen de la cloaca y endoscopía para observar las gónadas (Snyder et al. 1987). Entre los métodos no invasivos se encuentra la medición de niveles de esteroides en sangre o excremento y el análisis de ADN a través de plumas o de sangre (Ciembor et al. 2003, Romagno 2005). Sin embargo, estos métodos tienen un riesgo latente sobre la vida del animal o un costo económico alto, por lo que son relativamente poco prácticos para estudios de investigación y conservación en individuos de vida libre, más aún ante la carencia de recursos económicos y urgencia en la toma de decisiones. En cautiverio, la determinación del sexo cobra especial importancia para reunir parejas para su manejo en la reproducción.

Por estas razones, se han tratado de encontrar métodos alternativos para determinar el sexo en algunas especies a través de características morfológicas (Ciembor et al. 2003). Se busca con ello tener una herramienta útil, accesible y barata, sobre todo para la investigación y conservación en vida libre, y que no represente riesgo alguno para la población en estudio. Los análisis morfométricos han sido utilizados con éxito para definir dimorfismo sexual en algunas especies de aves donde el dimorfismo no es muy aparente. Ejemplos de esta utilidad existen para familias como los hirundí- 
nidos (De Lope 1985), los córvidos (Álvarez \& Aguilera 1985) y los colúmbidos (Favero 2001).

Para los psitácidos algunas de las características empleadas para la determinación del sexo, son el tamaño de la cabeza o del pico, el tamaño del ave, el color del iris y de las plumas, la abertura del hueso del pubis o el comportamiento agresivo (Snyder et al. 1987, Romagno 2005). Se incluye la presencia de colores más brillantes en las hembras en el caso del Kananga (Eclectus roratus; Heinsohn et al. 2005); o el color de las plumas bajo la cola en el loro de Senegal (Poicephalus senegalus; Sheasby \& Pattison 2003). El largo del pico es indicador sexual en las keas (Nestor notabilis; Bond et al. 1991) y su color lo es en las cacatúas negras (Calyptorhynchus sp.; Forshaw, 1989); por otro lado, el color del iris en cacatúas mayores de tres años indica el sexo, aunque estas técnicas no son completamente confiables (Bates \& Busenbark 1978, Lantermann 2000).

El desarrollo de métodos de este tipo no han sido aún explorados para la guacamaya verde Ara militaris. Aunque se conocen algunas de sus características generales que diferencian los sexos, como el tamaño y comportamiento (Forshaw 1989, IñigoElías 2000), dichas características son en ocasiones ambiguas o difíciles de observar, por lo que sólo se les considera indicadores para la determinación del sexo pero sin tener una certeza confiable. Entonces en general, para la guacamaya verde a la fecha no se ha logrado encontrar ningún carácter físico que defina un dimorfismo sexual (Juniper \& Parr 1998, Iñigo-Elías 2000). No obstante, a partir de la experiencia de campo de los estudios de comportamiento y de reproducción realizados en una población del estado de Oaxaca (Bonilla et al. 2007a, 2007b, Martínez \& Bonilla 2008), se presenta en este trabajo la hipótesis de la existencia de diferencias sexuales morfológicas en la especie que pueden ser observables en la forma, tamaño y proporciones de ciertas partes de la cabeza. Un indicador de tal naturaleza sería de gran utilidad durante las actividades de monitoreo y estudio de la biología de poblaciones silvestres, así como en la toma de decisiones para su manejo y conservación.

\section{MÉTODOS}

Dada la dificultad de emplear cualquier método que implique la captura de ejemplares silvestres de la guacamaya verde por lo inaccesible de los sitios de descanso y percha y por lo complejo que suele ser el obtener los permisos oficiales y de la comunidad para este tipo de manipulaciones, es importante volver a remarcar la utilidad de emplear un método, de preferencia que no implique el manejo invasivo de los ejemplares, que apoye las actividades de monitoreo y manejo de poblaciones silvestres. Se desarrolló un estudio morfométrico tendiente a conocer las características físicas que puedan reflejar el dimorfismo sexual en la guacamaya verde. Para ello, se utilizó una población cautiva de Ara militaris constituida por 18 ejemplares adultos (propie- 
dad de la organización civil denominada Aves del Paraíso y Criaturas Maravillosas A.C., ubicada en Puerto Vallarta, Jalisco, México). La organización civil encargó a laboratorios especializados en EUA que realizaran a 15 de estos ejemplares de guacamaya verde el análisis de sexado mediante pruebas de ADN. Se determinaron de esta forma, 11 hembras y cuatro machos, quedando tres individuos a los que no se les realizó el análisis de ADN por haber sido ingresados a la población cautiva en fecha posterior a los análisis. Esta determinación que se hizo del sexo de las guacamayas a través de ADN fue posteriormente corroborada a través de la observación de los individuos determinando los patrones de comportamiento observados en los individuos solitarios y en las parejas formadas. Algunas de estas parejas se encuentran a la fecha en un programa de reproducción. Es decir, el sexo determinado por análisis de ADN fue posteriormente corroborado por observaciones del comportamiento y formación de parejas por lo que tenemos la certeza de la definición del sexo de los individuos.

Con fundamento en experiencias previas (Bonilla et al. 2007a, 2007b, Martínez \& Bonilla 2008) y teniendo como hipótesis que algunas de las diferencias sexuales morfológicas se encuentran en la forma de la cabeza y en el tamaño y disposición de las diferentes estructuras observables en su perfil, se propone obtener un total de 11 medidas lineales a partir de fotografías de perfil de la cabeza de cada ejemplar; tres de ellas fueron determinadas a partir de dos líneas imaginarias pero identificables y trazables (Fig. 1a). El trabajar con fotografías probó ser un método eficaz, sin generar problemas para la medición de las medidas lineales de la cabeza. El tomar estas medidas sobre el animal vivo implicaría un manejo que estresa al animal y que hace muy complicado que la toma de las medidas sea estandarizada, con lo que se provocarían sesgos mayores que con las fotografías, que son fijas. Las fotografías han de tomarse de perfil, en un plano lo más transversal posible con respecto al plano de la cara. Debe apreciarse claramente el ojo, el pico y el perfil de la cabeza completa, así como el parche facial sin plumas (Fig. 1). De preferencia no debe utilizarse la foto si ésta fue tomada durante alguna vocalización que implique un movimiento significativo del pico.

En la elección de las medidas se tomaron en cuenta puntos sobre la estructura facial que fueran claramente identificables, como el culmen, la punta del pico, el centro del ojo y la escotadura posterior del parche facial. La medidas empleadas fueron 1) Longitud del pico (B), que es la distancia del culmen al extremo de la maxila; 2) Distancia entre el extremo del pico (Maxila) y la coronilla, pasando por el centro del ojo (BC2); 3) Distancia entre el culmen y la nuca, pasando por el centro del ojo (CN1); 4) Distancia entre el culmen y la nuca, pasando por la escotadura del parche facial (CN2); 5) Distancia entre el origen de la mandíbula a la coronilla pasando por el centro del ojo (ChC1) que se descompone en 6) Distancia entre el origen de la mandíbula al centro del Ojo (ChO); 7) Distancia entre el centro del ojo a la coronilla (OC1); la línea entre el origen de la mandíbula a la coronilla siguiendo la parte posterior del 

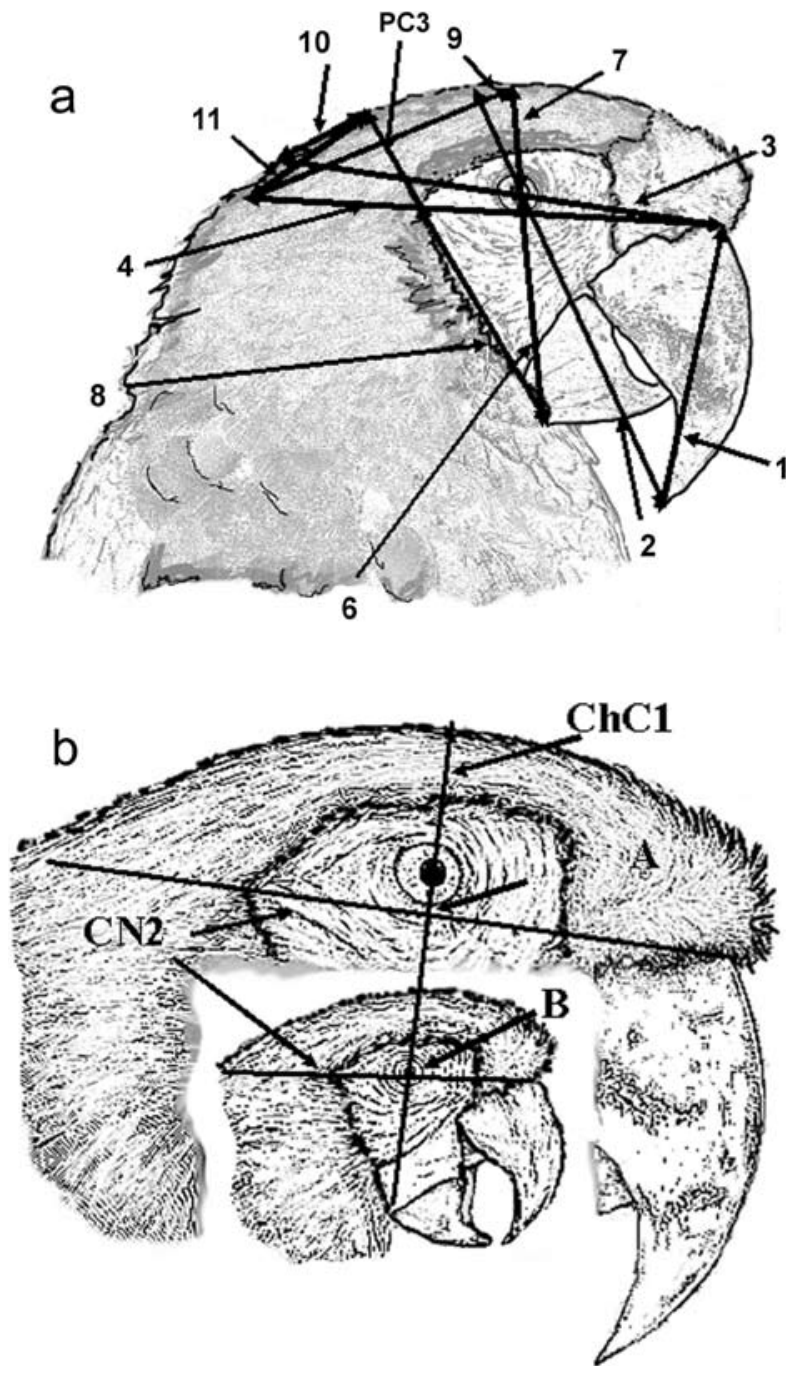

Figura 1. a) Medidas tomadas en las fotografías de cada ejemplar 1(B) Longitud del pico; 2) BC2 pico-coronilla 2; 3(CN1), Culmen-nuca $1 ; 4(\mathrm{CN} 2)$, culmen nuca 2; 5) ChC1 no está ilustrada y es la suma de las medidas 6 y 7; 6) ChO garganta-ojo; 7) OC1 Ojo coronilla 1; 8) ChP garganta parche; 9) C1N2 Coronilla1 nuca 2; 10 C3N1 Coronilla3 nuca 1; 11) C3N2 Coronilla 3 nuca 2; PC3 Parche coronilla 3, esta medida no fue obtenida, solo sirvió para obtener el punto de referencia para generar las medidas 10 y 11. b) Obtención del indicador de dimorfismo sexual en Ara militaris. La distancia que existe entre la línea CN2 y el borde inferior del ojo. A) El borde inferior del ojo presenta una posición elevada y el indicador adopta valores negativos, correspondiendo a una hembra. B) el borde inferior del ojo presenta una posición inferior y el indicador adopta valores positivos, correspondiendo a un macho. 
parche facial (ChC3) permite obtener 8) Distancia entre el origen de la mandíbula a la escotadura del parche facial (ChP); 9) distancia entre C1 y N2 (C1N2); 10) Distancia entre C3 y N1 (C3N1); y 11) Distancia entre C3 y N2 (C3N2). Todas las medidas fueron expresadas como una proporción de la distancia del culmen a la nuca pasando por la escotadura posterior del parche facial en las fotografías de cada ejemplar (medida 4, Fig. 1a). De esta forma se obtuvo una matriz de datos donde la magnitud de cada dato es una proporción de la medida 4.

Para evaluar la variación en cada una de las variables de acuerdo al sexo, se utilizaron los datos de los ejemplares con sexo conocido. Dado el tamaño muestral pequeño y desigual (cuatro machos y once hembras) se utilizó la prueba no paramétrica de Wilcoxon, considerando una significancia menor o igual a 0.05 . Se eligió ésta prueba porque no supone una distribución de tipo normal de los valores, que sería muy difícil de observar particularmente en los valores de los machos por la muestra tan pequeña. Para explorar las diferencias entre variables de los grupos se realizó un Análisis de Componentes Principales a partir de una matriz de varianza-covarianza. Dado que los datos están expresados como proporciones de la medida 4, la magnitud es similar, por lo que no se estandarizaron. El análisis estadístico se llevó a cabo utilizando el programa NCSS 2004 (Hintze 2004). Aunque otros métodos como el de Funciones Discriminantes parecen ser una mejor opción para maximizar las diferencias entre sexos, requieren de un tamaño de muestra mayor del que se disponía para este estudio, dado que es necesario usar solo una parte de los datos para generar las funciones discriminantes (entre el 25 y el $30 \%$ de los datos) y el resto para validarla (Huberty \& Olejnik 2006). El tipo de enfoque que usamos en el análisis permite probar de forma independiente la concordancia entre el resultado de la determinación del sexo a partir del ADN y las características morfométricas.

\section{RESULTADOS}

Las comparaciones univariadas mostraron diferencias significativas en los valores de las medidas 3 y 7 , que son medidas que tienen como referencia el centro del ojo; en ambas variables los machos tienen valores promedio más altos que las hembras (Cuadro 1). En el contexto multivariado, los dos primeros Componentes Principales explicaron el $82.5 \%$ de la varianza total de la muestra, con los machos mostrando muy poca variación en el Componente Principal 1 y valores extremos en el 2; no fue posible distinguir con certeza entre machos y hembras (Fig. 2). Las medidas que presentan cierta relevancia en el Componente Principal 1, son la 1, 2, 10 y 11 (Cuadro 1; Fig. 2). Las primeras dos variables tienen que ver con el tamaño y aparentemente con la forma del pico y su relación con la cabeza; por su parte, las otras dos variables tienen que ver con la forma del parche facial y la posición del ojo en el mismo. En el componente principal 2, las características relevantes fueron las 3, 6, 7 y 8 (Cua- 
Cuadro 1. Media y desviación estándar (entre paréntesis) de diez caracteres y resumen del Análisis de Componentes Principales. * = Medias entre sexos estadísticamente diferentes (Prueba de Wilcoxon, $p \leq 0.05$ ).

\begin{tabular}{lcccc}
\hline \multirow{2}{*}{ Variable } & \multicolumn{2}{c}{ Sexo } & \multicolumn{2}{c}{ Eigenvectores } \\
\cline { 2 - 5 } & Hembras & Machos & 0.3269 & PC2 \\
\hline B & $0.584(0.077)$ & $0.579(0.034)$ & 0.3963 & 0.3179 \\
BC2 & $0.918(0.091)$ & $0.943(0.070)$ & 0.1685 & 0.3580 \\
ChO & $0.484(0.058)$ & $0.481(0.026)$ & -0.0130 & 0.0017 \\
OC1* & $0.215(0.027)$ & $0.250(0.025)$ & 0.1551 & 0.3597 \\
ChC1 & $0.699(0.048)$ & $0.731(0.043)$ & 0.0950 & 0.5855 \\
ChP & $0.694(0.037)$ & $0.689(0.111)$ & -0.0077 & -0.0776 \\
CN1* & $0.864(0.041)$ & $0.947(0.016)$ & -0.3464 & 0.3457 \\
C1N2 & $0.675(0.088)$ & $0.662(0.065)$ & -0.4984 & 0.2870 \\
C3N2 & $0.389(0.117)$ & $0.398(0.045)$ & -0.5530 & 0.1981 \\
C3N1 & $0.204(0.120)$ & $0.320(0.059)$ & 67.76 & 14.77 \\
\% Var Expl & - & - & 67.76 & 82.54 \\
\% Var Expl Acum & - & - & &
\end{tabular}

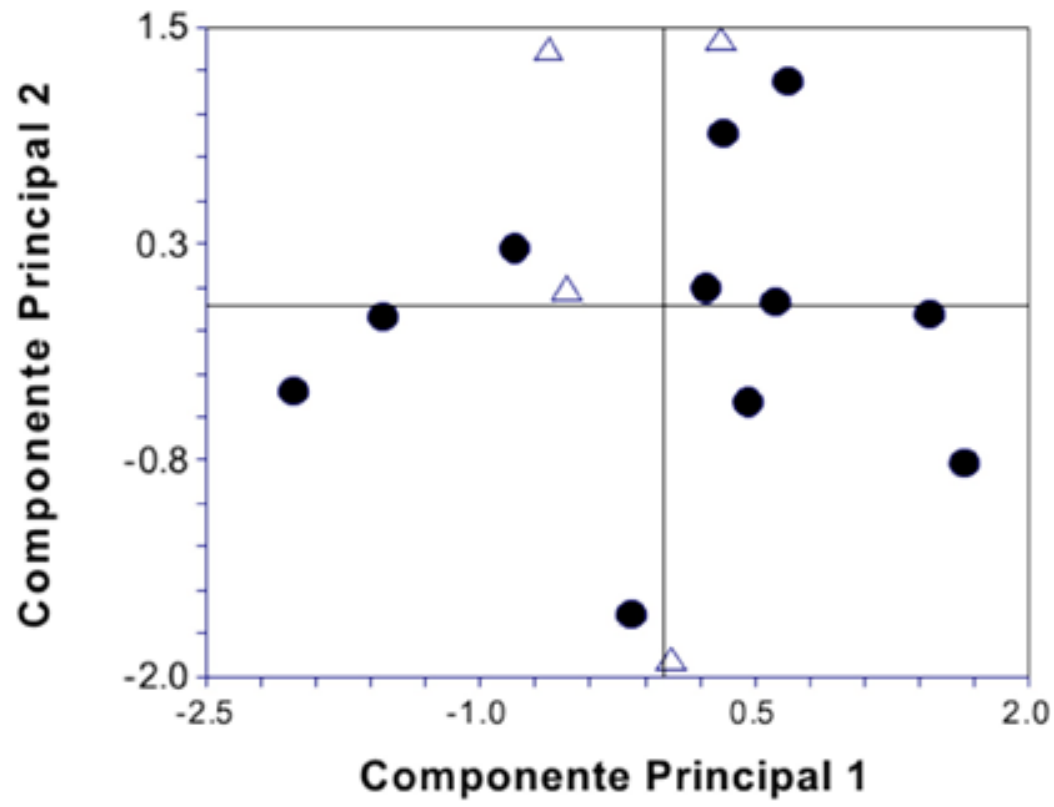

Figura 2. Proyección de 15 individuos de guacamaya verde de sexo conocido en los dos primeros Componentes Principales (Círculos $=$ Hembras, Triángulos $=$ Machos $)$. 
dro 1; Fig. 2). Estas variables tienen que ver con la posición del ojo y con la forma del parche facial. De esta forma, tanto la prueba de Wilcoxon como el Análisis de Componentes Principales sugirieron a la posición del ojo y la forma del parche facial como características importantes en el dimorfismo sexual de esta especie.

Con estos resultados, se buscó una forma de relacionarlos a fin de obtener un indicador complementario para la determinación del sexo. Para obtener este indicador, se empleó un Índice Ocular (IO), definido por la distancia entre la línea que define la medida $4(\mathrm{CN} 2)$ y el borde inferior del ojo, expresada ésta como un porcentaje del diámetro ocular:

$$
\mathrm{IO}=\left(\mathrm{D}_{(\mathrm{CN} 2-\mathrm{O})} / \mathrm{DO}\right) * 100
$$

Donde: $\mathrm{D}_{(\mathrm{CN} 2-\mathrm{O})}=$ Distancia entre $\mathrm{CN} 2$ y el borde inferior del ojo

Donde: $\mathrm{DO}=$ Diámetro ocular

El valor que resulta puede ser positivo si el borde inferior del ojo se encuentra por debajo de la línea CN2 (Fig. 1b B), o negativo cuando el borde inferior del ojo se encuentra arriba de dicha línea (Fig. 1b A).

Las hembras tendieron a presentar el ojo en una posición más elevada, por lo que el valor del indicador propuesto presentó principalmente valores negativos $(\bar{x}=$ -12.74 , desviación estándar $\mathrm{SD}=15.82$ ). Por el contrario, los machos tendieron a presentar valores positivos de este indicador $(\bar{x}=27.39, \mathrm{SD}=9.86)$. Estas diferencias fueron significativas (prueba de Wilcoxon: $\mathrm{W}=66 ; \mathrm{P}=0.005$ ).

Al tomar como referencia a los tres ejemplares cuyo sexo era desconocido, dos fueron identificados como hembras ( $\mathrm{IO}=0$ en ambos casos); el tercero $(\mathrm{IO}=13.165)$ no pudo ser definido claramente por este medio (Fig. 3). Sin embargo, al observar los valores para la medidas 3 y 6 de este individuo ( 0.929 y 0.205 , respectivamente), éstas quedan incluidas principalmente dentro de las esperadas para las hembras, por lo que tentativamente estos ejemplares pueden ser considerados como miembros de este sexo, hasta que estudios moleculares o comportamentales lo comprueben.

\section{DISCUSIÓN}

El escaso número de ejemplares con los que se contó para realizar el presente estudio revela las dificultades para estudiar esta especie. A pesar de que se revisaron incluso ejemplares de colecciones como la Colección Nacional de Aves de la colección de la Escuela Nacional de Ciencias Biológicas y la colección osteológica del Instituto Nacional de Antropología e Historia, no se pudieron obtener datos de ejemplares debidamente sexados.

Aunque no fue posible separar claramente a los dos sexos a través del análisis multivariado de la información morfométrica, al analizar el comportamiento de los valores que toman cada una de las variables en los dos primeros componentes, se 


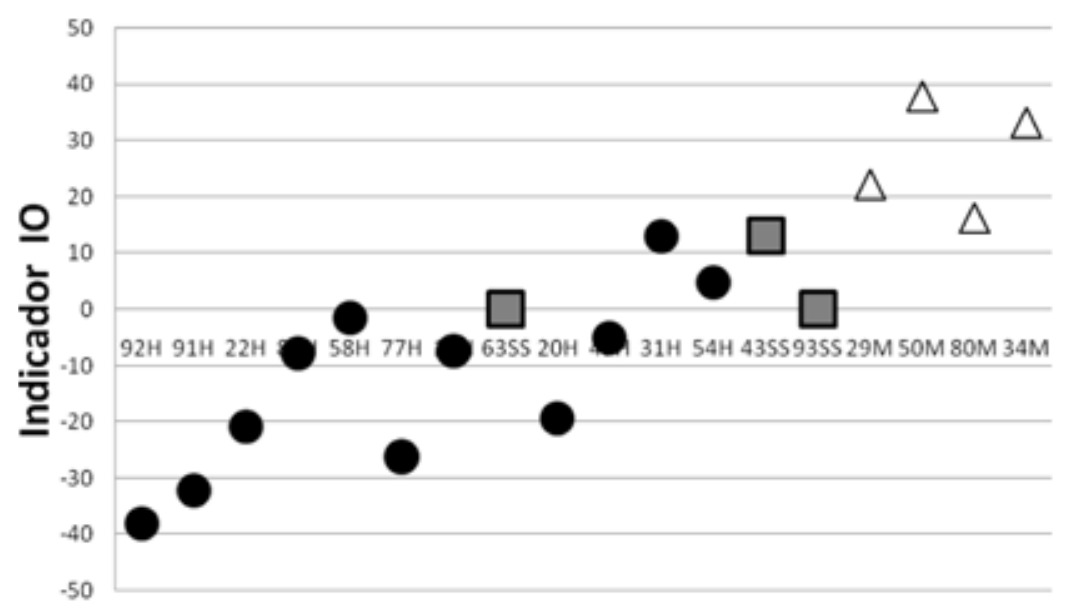

Ejemplar

Figura 3. Relación del indicador propuesto con el sexo determinado por medio de ADN. H) Hembra; M) Macho; SS) Sexo desconocido.

reafirma la relevancia de algunas características en la identificación del sexo. Particularmente, fueron importantes la forma y disposición del parche facial puesto que la escotadura posterior de éste se tomó como referencia en la obtención de algunas medidas (como la 4 y la 8), y afectan directamente a otras (como la 10 y la 11) que resultaron ser relevantes en el análisis multivariado. La forma típica de una hembra presenta un parche más amplio, cuyo borde posterior define una línea que señala imaginariamente hacia la nuca del ejemplar. Esto es, la parte de la cabeza posterior al parche facial es proporcionalmente mayor en el macho, lo que da la apariencia de tener la cabeza más cuadrangular que esférica. Con estos resultados a la mano, estadísticamente se puede afirmar que existe una diferencia intersexual relacionada con la posición del ojo en la cabeza y el tamaño y forma del parche facial.

Por otro lado y dado que el estudio de un posible crecimiento alométrico en psitácidos y en especial en Ara militaris son muy escasos o no existen, no se tiene certeza de que las proporciones entre las medidas empleadas no tengan alguna variación con la edad, por lo que el utilizar individuos adultos previene cualquier sesgo debido a esta situación.

La distinción entre sexos de los ejemplares por medio del Índice Ocular es mucho más simple y clara. Esta variable representa un método que define claramente en la mayoría de los casos el sexo de los individuos. Una combinación de ambos análisis (PCA e IO) podría resolver un porcentaje próximo al 100\% de los casos y convertirse en un método no invasivo de determinación del sexo en Ara militaris, sin riesgos y 
mucho más económico que los empleados actualmente (Cuadro 2). Es importante aclarar el valor de esta herramienta para estudios de campo y manejo de poblaciones silvestres. Se propone que se revise la utilidad de este índice para otras especies de Psitacidae. Para el manejo de ejemplares en cautividad, donde la certidumbre en la determinación del sexo es vital para los programas de reproducción, probablemente sea mejor usar el método de determinación del sexo por ADN, pues su mayor certeza compensa el mayor costo del análisis.

AGRADECIMIENTOS. Se agradecen las facilidades en la obtención del material fotográfico y los datos de sexo de los ejemplares a Aves del paraíso y Criaturas Maravillosas A.C. A Parrots International por el apoyo financiero que apoyo parcialmente la realización de este trabajo y al Instituto Politécnico Nacional por el apoyo recibido a través del proyecto "Hábitos alimentarios durante la temporada de empollamiento en dos poblaciones de Ara militaris en México" clave 20080402. Se agradece a dos revisores anónimos cuyos comentarios mejoraron sustancialmente nuestro manuscrito.

Cuadro 2. Comparativa de determinación de sexo por medio de los tres métodos: análisis de ADN $(\mathrm{ADN})$, análisis de componentes principales (PCA) e Índice Ocular (IO). Se presentan las medidas más significativas de acuerdo a la prueba de Wilcoxon y el valor del índice ocular (VIO).

\begin{tabular}{ccccccc}
\hline Ejemplar & $7(\mathrm{OC} 1)$ & \multirow{2}{*}{$3(\mathrm{CN} 1)$} & $\mathrm{VIO}$ & \multicolumn{3}{c}{ Determinación de sexo } \\
\cline { 5 - 6 } & & & & $\mathrm{ADN}$ & $\mathrm{PCA}$ & $\mathrm{IO}$ \\
\hline 92 & 0.191319 & 0.821932 & -37.9457 & $\mathrm{H}$ & $\mathrm{H}$ & $\mathrm{H}$ \\
91 & 0.199193 & 0.817366 & -32.1317 & $\mathrm{H}$ & $\mathrm{H}$ & $\mathrm{H}$ \\
77 & 0.269083 & 0.833687 & -26.1169 & $\mathrm{H}$ & - & $\mathrm{H}$ \\
22 & 0.196084 & 0.845148 & -20.7301 & $\mathrm{H}$ & $\mathrm{H}$ & $\mathrm{H}$ \\
20 & 0.229202 & 0.901143 & -19.3026 & $\mathrm{H}$ & $\mathrm{H}$ & $\mathrm{H}$ \\
87 & 0.176053 & 0.837926 & -7.7383 & $\mathrm{H}$ & $\mathrm{H}$ & $\mathrm{H}$ \\
19 & 0.22324 & 0.868292 & -7.4203 & $\mathrm{H}$ & $\mathrm{H}$ & $\mathrm{H}$ \\
45 & 0.249197 & 0.899207 & -5.0889 & $\mathrm{H}$ & - & $\mathrm{H}$ \\
58 & 0.204472 & 0.83308 & -1.4595 & $\mathrm{H}$ & $\mathrm{H}$ & $\mathrm{H}$ \\
54 & 0.224068 & 0.919179 & 4.8292 & $\mathrm{H}$ & $\mathrm{H}$ & $\mathrm{H}$ \\
31 & 0.205045 & 0.929552 & 12.967 & $\mathrm{H}$ & $\mathrm{H}$ & $\mathrm{H}$ \\
80 & 0.285082 & 0.933743 & 16.316 & $\mathrm{M}$ & $\mathrm{M}$ & $\mathrm{M}$ \\
29 & 0.225626 & 0.936163 & 22.22 & $\mathrm{M}$ & - & $\mathrm{M}$ \\
34 & 0.251832 & 0.969591 & 33.1853 & $\mathrm{M}$ & $\mathrm{M}$ & $\mathrm{M}$ \\
50 & 0.238172 & 0.951306 & 37.8317 & $\mathrm{M}$ & - & $\mathrm{M}$ \\
63 & 0.191716 & 0.896124 & 0 & $\mathrm{SS}$ & $\mathrm{H}$ & $\mathrm{H}$ \\
93 & 0.230866 & 0.93862 & 0 & $\mathrm{SS}$ & - & $\mathrm{H}$ \\
43 & 0.219819 & 0.916478 & 13.165 & $\mathrm{SS}$ & $\mathrm{H}$ & - \\
\hline
\end{tabular}




\section{LITERATURA CITADA}

Álvarez, F. \& E. Aguilera. 1988. Sobre el dimorfismo sexual en el rabilargo, Cyanopyca cyanea Pall. Ardeola, 35: 269-295.

Bates, H. \& R. L. Busenbark. 1978. Parrots and related birds. THF Publisher. New Jersey.

Bond, A. B., K. J. Wilson \& J. Diamond. 1991. Sexual dimorphism in the kea Nestor notabilis. EMU, 91: 12-19.

Bonilla, R. C., G. Reyes M. \& L. Santiago C. 2007a. Ámbito hogareño de la guacamaya verde (Ara militaris) en la cañada Oaxaqueña. Mesoamericana, 11: 54-61.

Bonilla, R. C., G. Reyes M. \& R. García. 2007b. Observations of the military macaw (Ara militaris) in Northern Oaxaca, Mexico. Wilson Journal of Ornithology, 119: 731-734.

Ciembor, P., M. J. Murray, C. R. Gregory, B. L Speer, D. J. Harris, M. Taylor \& B. W. Ritchie. 2003. Sex determination in Psittaciformes. Convention Proceedings of the International Aviculturist's Society. Memphis, TN, U.S.A.

De Lope, F. 1985. Pterilosis y dimorfismo sexual de Hirundo rustica rustica L. en Extremadura (España). Ardeola, 32: 3-8.

Favero, M. 2001. Características morfológicas y dimorfismo sexual en la paloma antártica (Chionis alba). Ornitologia Neotropical, 12: 173-179.

Forshaw, J. M., 1989. Parrots of the world. Third ed. Landsdowne Editions, Melbourne, Australia.

Heinsohn R., S. Legge \& J. A. Endler. 2005. Extreme reversed sexual dichromatism in a bird without sex role reversal. Science, 309: 617-619.

Hintze, J. 2004. NCSS and Pass. Number cruncher statistical system. Kaysvillr, Utah. www.ncss.com.

Huberty, C. J. \& S. Olejnik. 2006. Applied MANOVA and discriminant analysis. 2d. ed. Wiley Intercience. Hoboken, New Jersey.

Iñigo-Elías, E. 2000. Guacamaya verde (Ara militaris). Pp. 213-215. In: Ceballos, G. y V. L. Márquez (Coord.). Las aves de México en peligro de extinción. $1^{\mathrm{a}}$ ed. Fondo de Cultura Económica, México.

Juniper, T. \& M. Parr. 1998. Parrots. A guide to parrots of the world. Yale University Press, U.S.A.

Lantermann, W. 2000. Cockatoos: A complete pet owner's manual. Barron's Educational Series. NY, U.S.A.

Martínez, D. R. \& C. Bonilla Ruz. 2008. Hábitos alimenticios de Ara militaris en la reserva de la biosfera Tehuacán-Cuicatlán, México. Mesoamericana, 11: 45-50.

Romagno, A. 2005. Reproduction and paediatrics, pp. 222-233. In: Harcourt-Brown, N. and J. Chitty (Eds.). BSAVA Manual of psitacine birds. British Small Animal Veterinary Association. Wiley eds. NJ.

Santos, S. I., B. Elward \& J. T. Lumeij. 2006. Sexual dichromatism in the Blue-fronted Amazon Parrot (Amazona aestiva) revealed by multiple-angle Spectrometry. Journal of Avian Medicine and Surgery, 20: 8-14.

Sheasby, R. \& J. Pattison. 2003. Visual sexing of Senegals. www.wingscc.com/aps/apsmail/senegal_ sexing.aspx.

Snyder, N. F. R., J. W. Wiley \& C. B. Kepler. 1987. The parrots of Luquillo: Natural History and conservation of the Puerto Rican parrot. Western Fundation of Vertebrate Zoology. Los Angeles, CA. 